Bull. Korean Math. Soc. 52 (2015), No. 1, pp. 263-273

http://dx.doi.org/10.4134/BKMS.2015.52.1.263

\title{
ON EVALUATIONS OF THE MODULAR $j$-INVARIANT BY MODULAR EQUATIONS OF DEGREE 2
}

\author{
Dae Hyun Paek ${ }^{\dagger}$ and Jinhee Yi
}

\begin{abstract}
We derive modular equations of degree 2 to establish explicit relations for the parameterizations for the theta functions $\varphi$ and $\psi$. We then find specific values of the parameterizations to evaluate some new values of the modular $j$-invariant in terms of $J_{n}$.
\end{abstract}

\section{Introduction}

The invariants $J(\tau)$ and $j(\tau)$, for $\tau \in \mathbb{H}=\{\tau: \operatorname{Im} \tau>0\}$, are defined by

$$
J(\tau)=\frac{g_{2}^{3}(\tau)}{\Delta(\tau)} \quad \text { and } \quad j(\tau)=1728 J(\tau)
$$

where,

$$
\begin{gathered}
\Delta(\tau)=g_{2}^{3}(\tau)-27 g_{3}^{2}(\tau), \\
g_{2}(\tau)=60 \sum_{\substack{m, n=-\infty \\
(m, n) \neq(0,0)}}^{\infty}(m \tau+n)^{-4},
\end{gathered}
$$

and

$$
g_{3}(\tau)=140 \sum_{\substack{m, n=-\infty \\(m, n) \neq(0,0)}}^{\infty}(m \tau+n)^{-6} .
$$

Moreover, the function $\gamma_{2}(\tau)$ is defined by $([5, \mathrm{p} .249])$

$$
\gamma_{2}(\tau)=\sqrt[3]{j(\tau)}
$$

where the principal branch is chosen. Ramanujan defined a function $J_{n}$ by

$$
J_{n}=\frac{1-16 \alpha_{n}\left(1-\alpha_{n}\right)}{8\left(4 \alpha_{n}\left(1-\alpha_{n}\right)\right)^{1 / 3}},
$$

Received January 11, 2014; Revised July 7, 2014.

2010 Mathematics Subject Classification. Primary 11F27, 11R29, 33C90; Secondary 33C05, 33E05.

Key words and phrases. theta functions, modular equations, modular $j$-invariant.

$\dagger$ This work is supported by the Education Research Institute, Busan National University of Education in 2014. 
where $\alpha_{n}=k_{n}^{2}$ and $n$ is a natural number. Here, as usual, in the theory of elliptic functions, let $k, 0<k<1$, denote the modulus, then the singular modulus $k_{n}$ is defined by $k_{n}=k\left(e^{-\pi \sqrt{n}}\right)$. To identify $J_{n}$ with the class invariant $G_{n}$, we first, as usual, set

$$
(a ; q)_{\infty}=\prod_{k=0}^{\infty}\left(1-a q^{k}\right), \quad|q|<1,
$$

and let

$$
\chi(q)=\left(-q ; q^{2}\right)_{\infty} .
$$

Furthermore, for $|a b|<1$, Ramanujan's general theta function $f(a, b)$ is defined by

$$
f(a, b)=\sum_{n=-\infty}^{\infty} a^{n(n+1) / 2} b^{n(n-1) / 2} .
$$

Then, the classical theta functions $\varphi, \psi$, and $f$ are defined by, for $|q|<1$,

$$
\begin{gathered}
\varphi(q)=f(q, q)=\sum_{n=-\infty}^{\infty} q^{n^{2}}=\left(-q ; q^{2}\right)_{\infty}^{2}\left(q^{2} ; q^{2}\right)_{\infty}=\frac{(-q ;-q)_{\infty}}{(q ;-q)_{\infty}} \\
\psi(q)=f\left(q, q^{3}\right)=\sum_{n=0}^{\infty} q^{n(n+1) / 2}=\frac{\left(q^{2} ; q^{2}\right)_{\infty}}{\left(q ; q^{2}\right)_{\infty}},
\end{gathered}
$$

and

$$
f(-q)=f\left(-q,-q^{2}\right)=\sum_{n=-\infty}^{\infty}(-1)^{n} q^{n(3 n-1) / 2}=(q ; q)_{\infty} .
$$

From $(q ; q)_{\infty}=\left(q ; q^{2}\right)_{\infty}\left(q^{2} ; q^{2}\right)_{\infty}$, it is easily seen that

$$
\chi(-q)=\frac{f(-q)}{f\left(-q^{2}\right)} .
$$

If $q_{n}=e^{-\pi \sqrt{n}}$ and $n$ is a positive rational number, then the class invariant $G_{n}$ is defined by

$$
G_{n}=2^{-1 / 4} q_{n}^{-1 / 24} \chi\left(q_{n}\right)
$$

Since, by [2, p. 124],

$$
\chi(q)=2^{1 / 6}\left(\frac{q}{\alpha(1-\alpha)}\right)^{1 / 24}
$$

it follows from (1.4) that

$$
G_{n}=\left(4 \alpha_{n}\left(1-\alpha_{n}\right)\right)^{-1 / 24} .
$$

Hence, by (1.2) and (1.5), we find that

$$
J_{n}=\frac{1}{8} G_{n}^{8}\left(1-4 G_{n}^{-24}\right) .
$$


We now identify $J_{n}$ with $\gamma_{2}$. From [5, Theorem 12.17], for $q=e^{2 \pi i \tau}$,

$$
\gamma_{2}(\tau)=2^{8} \frac{q^{2 / 3} f^{16}\left(-q^{2}\right)}{f^{16}(-q)}+\frac{f^{8}(-q)}{q^{1 / 3} f^{8}\left(-q^{2}\right)} .
$$

Setting $\tau=\frac{3+\sqrt{-n}}{2}$, we deduce from (1.3), (1.4), and (1.7) that

$$
\gamma_{2}\left(\frac{3+\sqrt{-n}}{2}\right)=-4 G_{n}^{8}\left(1-4 G_{n}^{-24}\right) .
$$

Hence, by (1.1) and (1.6), we have

$$
J_{n}=-\frac{1}{32} \gamma_{2}\left(\frac{3+\sqrt{-n}}{2}\right)=-\frac{1}{32} \sqrt[3]{j\left(\frac{3+\sqrt{-n}}{2}\right)} .
$$

Therefore, in general, the value of $J_{n}$ can be obtained in terms of $G_{n}$ or $j\left(\frac{3+\sqrt{-n}}{2}\right)$ for any natural number $n$. For 15 values of $n$ such that $n \equiv 3$ ( $\bmod 4)$, Ramanujan indicated the corresponding 15 values of $J_{n}$, although some are not given very explicitly. There are 13 cases when the class number of the order in an imaginary quadratic fields equals 1 ([5, p. 260]). In such an instance, the value of $j$-invariant is known to be an integer. In these cases, Ramanujan gave 7 values of $J_{n}$ for $n=3,11,19,27,43,67$, and 163 . See [1, pp. 310-311] for more details. Formula (1.6) can be used to evaluate some of these values such as $J_{3}=0$ and $J_{27}=5 \cdot 3^{1 / 3}$, but in most instances the value of $G_{n}$ is unavailable. For the rest of cases of degree 1 , the values of $J_{n}$ can be evaluated by using the relation (1.8) and the corresponding $j$-invariant given in [5, p. 261]. It is also known that there are 29 cases when the degree of $j\left(\frac{3+\sqrt{-n}}{2}\right)$ equals 2 . Ramanujan dealt with 6 of these: the values of $J_{n}$ for $n=35,51,75,91,99$, and 115 , even though he did not record the value of $J_{99}$ explicitly. See also [1, pp. 311-312] for more details.

As an instance of using the values of $J_{n}$, Ramanujan further defined a function $t_{n}$ by, for $q=e^{-\pi \sqrt{n}}$,

and asserted that

$$
t_{n}=\sqrt{3} q^{1 / 18} \frac{f\left(q^{1 / 3}\right) f\left(q^{3}\right)}{f^{2}(q)}
$$

$$
t_{n}=\left(2 \sqrt{64 J_{n}^{2}-24 J_{n}+9}-\left(16 J_{n}-3\right)\right)^{1 / 6} .
$$

Ramanujan then considered the polynomials $p_{n}(t)$ satisfied by $t_{n}$ for $n=$ $11,35,59,83$, and 107 . These polynomials are extremely simple, whereas the corresponding polynomials of the same degrees satisfied by $J_{n}$ are more complicated. Refer [4] to see that if $n$ is square free, $n \equiv 11(\bmod 24)$, and the class number of the Hilbert class field is odd, then $t_{n}$ and $J_{n}$ satisfy irreducible polynomials of the same degree.

Since modular equations are crucial in this study on evaluations of the modular $j$-invariant in terms of $J_{n}$, we now give a definition of a modular equation. 
Let $a, b$, and $c$ be arbitrary complex numbers except that $c$ cannot be a nonpositive integer. Then, for $|z|<1$, the Gaussian or ordinary hypergeometric function ${ }_{2} F_{1}(a, b ; c ; z)$ is defined by

$$
{ }_{2} F_{1}(a, b ; c ; z)=\sum_{n=0}^{\infty} \frac{(a)_{n}(b)_{n}}{(c)_{n} n !} z^{n},
$$

where $(a)_{0}=1$ and $(a)_{n}=a(a+1)(a+2) \cdots(a+n-1)$ for each positive integer $n$.

The complete elliptic integral of the first kind $K(k)$ is defined by

$$
K(k)=\int_{0}^{\pi / 2} \frac{d \theta}{\sqrt{1-k^{2} \sin ^{2} \theta}}=\frac{\pi}{2}{ }_{2} F_{1}\left(\frac{1}{2}, \frac{1}{2} ; 1 ; k^{2}\right)=\frac{\pi}{2} \varphi^{2}\left(e^{-\pi \frac{K^{\prime}}{K}}\right),
$$

where $0<k<1, K^{\prime}=K\left(k^{\prime}\right)$, and $k^{\prime}=\sqrt{1-k^{2}}$. The number $k$ is called the modulus of $K$ and $k^{\prime}$ is called the complementary modulus. Let $K, K^{\prime}, L$, and $L^{\prime}$ denote complete elliptic integrals of the first kind associated with the moduli $k, k^{\prime}, l$, and $l^{\prime}$, respectively, where $0<k<1$ and $0<l<1$. Suppose that

$$
\frac{L^{\prime}}{L}=n \frac{K^{\prime}}{K}
$$

holds for some positive integer $n$. Then a relation between $k$ and $l$ induced by (1.10) is called a modular equation of degree $n$. If we set

$$
q=e^{-\pi \frac{K^{\prime}}{K}} \quad \text { and } \quad q^{\prime}=e^{-\pi \frac{L^{\prime}}{L}},
$$

then (1.10) is equivalent to the relation $q^{n}=q^{\prime}$. Hence a modular equation can be viewed as an identity involving theta functions at the arguments $q$ and $q^{n}$. Set $\alpha=k^{2}$ and $\beta=l^{2}$, then we say that $\beta$ has degree $n$ over $\alpha$.

We now turn to evaluations of $J_{n}$ by using a parametrization $r_{k, n}$ for the theta function $f$. In [6], $r_{k, n}$ is defined by, for any positive real numbers $k$ and $n$,

$$
r_{k, n}=\frac{f(-q)}{k^{1 / 4} q^{(k-1) / 24} f\left(-q^{k}\right)},
$$

where $q=e^{-2 \pi \sqrt{n / k}}$. For convenience, we write $r_{n}$ instead of $r_{2, n}$. Then, $G_{n}$ can be written in terms of $r_{n}([6$, Theorem 2.2.3])

$$
G_{n}=\frac{r_{2 n}}{2^{1 / 4} r_{n / 2}} .
$$

Hence (1.6) can also be written in terms of $r_{n}$

$$
J_{n}=\frac{1}{32}\left(\frac{r_{2 n}}{r_{n / 2}}\right)^{8}\left(1-2^{8}\left(\frac{r_{n / 2}}{r_{2 n}}\right)^{24}\right) .
$$


Using the formula (1.12), the explicit values of $J_{1}, J_{2}, \ldots, J_{10}$ were evaluated in [6]. In particular,

$$
\begin{gathered}
J_{2}=\frac{5}{16}(-19+13 \sqrt{2}), \\
J_{4}=\frac{3}{32}(-724+513 \sqrt{2}), \\
J_{8}=\frac{(1+\sqrt{2})^{3}(4+\sqrt{2+10 \sqrt{2}})^{3}-256}{32(1+\sqrt{2})^{2}(4+\sqrt{2+10 \sqrt{2}})^{2}} .
\end{gathered}
$$

Meanwhile the relation (1.6) can also be written in terms of parametrizations $h_{k, n}^{\prime}$ and $l_{k, n}^{\prime}$ for the theta function $\varphi$ and $\psi$, respectively. In $[7,8], h_{k, n}^{\prime}$ and $l_{k, n}^{\prime}$ are defined by, for any positive real numbers $k$ and $n$,

$$
h_{k, n}^{\prime}=\frac{\varphi(-q)}{k^{1 / 4} \varphi\left(-q^{k}\right)},
$$

where $q=e^{-2 \pi \sqrt{n / k}}$ and

$$
l_{k, n}^{\prime}=\frac{\psi(q)}{k^{1 / 4} q^{(k-1) / 8} \psi\left(q^{k}\right)},
$$

where $q=e^{-\pi \sqrt{n / k}}$. For convenience, we write $h_{n}^{\prime}$ and $l_{n}^{\prime}$ instead of $h_{2, n}^{\prime}$ and $l_{2, n}^{\prime}$, respectively, throughout this paper. Then it follows from [8, Theorem 6.3] that

$$
l_{2 n}^{\prime}=\frac{r_{2 n}^{2}}{r_{n / 2}} \quad \text { and } \quad h_{2 n}^{\prime}=\frac{r_{n / 2}^{2}}{r_{2 n}} .
$$

Hence (1.12) can be written in the alternative form

$$
J_{n}=\frac{1}{32}\left(\frac{l_{2 n}^{\prime}}{h_{n / 2}^{\prime}}\right)^{8 / 3}\left(1-2^{8}\left(\frac{h_{n / 2}^{\prime}}{l_{2 n}^{\prime}}\right)^{8}\right) \text {. }
$$

In addition, in [7], $h_{k, n}$ is defined by, for any positive real numbers $k$ and $n$,

$$
h_{k, n}=\frac{\varphi(q)}{k^{1 / 4} \varphi\left(q^{k}\right)},
$$

where $q=e^{-\pi \sqrt{n / k}}$. For convenience, we also write $h_{n}$ in stead of $h_{2, n}$ throughout this paper.

Note that specific values of $h_{n}$ will play crucial roles in evaluating the corresponding values of $h_{n}^{\prime}$ and $l_{n}^{\prime}$ later on. This study is motivated by the values of $J_{2}, J_{4}$, and $J_{8}$ obtained from (1.12). Some values of the modular $j$-invariant, as mentioned before, were used to evaluate the values of $J_{n}$ for $n=11,19,43,67$, and 163. Hence, instead of evaluating some values of the modular $j$-invariant to find the corresponding values of $J_{n}$, we first employ (1.13) to evaluate the 
values of $J_{n}$, and then find the corresponding values of $j\left(\frac{3+\sqrt{-n}}{2}\right)$ by the relation (1.8), in the case of when $n$ is of the form $n=2^{2 m-1}$ or $n=2^{2 m}$ for every positive integer $m$. Note that our results contain the values of $J_{2}, J_{4}$, and $J_{8}$ as special cases. In order to do so, we first derive modular equations of degree 2 for the theta functions $\varphi$ and $\psi$. We then find explicit relations for the corresponding parameterizations, evaluate some numerical values of $h_{n}, h_{n}^{\prime}$, and $l_{n}^{\prime}$, and evaluate some new values of $J_{n}$ so that we have the corresponding values of the modular $j$-invariant.

\section{Preliminary results}

In this section, we introduce fundamental theta function identities that will play key roles in deriving modular equations of degree 2 . Let $k$ be the modulus as in (1.9). Set $x=k^{2}$ and also set

$$
k^{2}=x=1-\frac{\varphi^{4}(-q)}{\varphi^{4}(q)} .
$$

Then

$$
\varphi^{2}(q)={ }_{2} F_{1}\left(\frac{1}{2}, \frac{1}{2} ; 1 ; x\right)=z
$$

where

$$
q=e^{-y}=\exp \left(-\pi \frac{{ }_{2} F_{1}\left(\frac{1}{2}, \frac{1}{2} ; 1 ; 1-x\right)}{{ }_{2} F_{1}\left(\frac{1}{2}, \frac{1}{2} ; 1 ; x\right)}\right)=\exp \left(-\pi \frac{K\left(k^{\prime}\right)}{K(k)}\right) .
$$

Lemma 2.1 ([3, Theorem 5.4.1]). If $x, q$, and $z$ are related by (2.1), (2.2), and (2.3), then
(i) $\varphi(q)=\sqrt{z}$,
(ii) $\varphi(-q)=\sqrt{z}(1-x)^{1 / 4}$,
(iii) $\varphi\left(q^{2}\right)=\sqrt{z} \sqrt{\frac{1+\sqrt{1-x}}{2}}$,
(iv) $\varphi\left(-q^{2}\right)=\sqrt{z}(1-x)^{1 / 8}$
(v) $\varphi\left(q^{4}\right)=\frac{1}{2} \sqrt{z}\left(1+(1-x)^{1 / 4}\right)$

Lemma 2.2 ([3, Theorem 5.4.2]). If $x, q$, and $z$ are related by (2.1), (2.2), and (2.3), then
(i) $\psi(q)=\sqrt{\frac{1}{2} z}\left(\frac{x}{q}\right)^{1 / 8}$,
(ii) $\psi\left(q^{2}\right)=\frac{1}{2} \sqrt{z}\left(\frac{x}{q}\right)^{1 / 4}$.

\section{Modular equations of degree 2}

In this section, we derive modular equations of degree 2 and establish some explicit relations for $h_{n}, h_{n}^{\prime}$, and $l_{n}^{\prime}$ for some positive real number $n$ by using these modular equations. 
Theorem 3.1. If $P=\frac{\varphi(q)}{\varphi\left(q^{2}\right)}$ and $Q=\frac{\varphi\left(q^{2}\right)}{\varphi\left(q^{4}\right)}$, then

$$
P Q+\frac{2}{P Q}=\frac{Q}{P}+2 \text {. }
$$

Proof. By Lemma 2.1(i), (iii), and (v),

$$
P=\sqrt{\frac{2}{1+\sqrt{1-\alpha}}} \text { and } Q=\frac{\sqrt{2} \sqrt{1+\sqrt{1-\alpha}}}{1+(1-\alpha)^{1 / 4}} .
$$

Combine and rearrange the last two equalities in terms of $P$ and $Q$ to complete the proof.

For a different proof of Theorem 3.1, see [7, Theorem 4.2]. Now, by the definition of $h_{n}$, we have:

Corollary 3.2. For every positive real number $n$, we have

$$
\sqrt{2}\left(h_{n} h_{4 n}+\frac{1}{h_{n} h_{4 n}}\right)=\frac{h_{4 n}}{h_{n}}+2 .
$$

Note that (3.2) is the same equation as in [7, Theorem 4.6].

Theorem 3.3. If $P=\frac{\varphi(q)}{\varphi\left(q^{2}\right)}$ and $Q=\frac{\varphi(-q)}{\varphi\left(-q^{2}\right)}$, then

$$
P^{2}\left(Q^{4}+1\right)=2 \text {. }
$$

Proof. By Lemma 2.1(i)-(iv),

$$
P=\sqrt{\frac{2}{1+\sqrt{1-\alpha}}} \text { and } Q=(1-\alpha)^{1 / 8} .
$$

Combining and rearranging the last two equalities in terms of $P$ and $Q$, we complete the proof.

By the definitions of $h_{n}$ and $h_{n}^{\prime}$, we have:

Corollary 3.4. For every positive real number $n$, we have

$$
h_{n}^{2}\left(2 h_{n / 4}^{\prime 4}+1\right)=\sqrt{2} .
$$

Theorem 3.5. If $P=\frac{\varphi(q)}{\varphi\left(q^{2}\right)}$ and $Q=\frac{\psi(q)}{q^{1 / 8} \psi\left(q^{2}\right)}$, then

$$
4 P^{4}=\left(P^{2}-1\right) Q^{8} .
$$

Proof. By Lemma 2.1(i) and (iii) and Lemma 2.2,

$$
P=\sqrt{\frac{2}{1+\sqrt{1-\alpha}}} \text { and } Q=\frac{\sqrt{2}}{\alpha^{1 / 8}} .
$$

Combining and rearranging the last two equalities in terms of $P$ and $Q$, we have the required result.

By the definitions of $h_{n}$ and $l_{n}^{\prime}$, we have: 
Corollary 3.6. For every positive real number $n$, we have

$$
2 h_{n}^{4}=\left(\sqrt{2} h_{n}^{2}-1\right) l_{n}^{\prime 8} .
$$

\section{Specific values of $h_{n}, h_{n}^{\prime}$, and $l_{n}^{\prime}$}

We are now in position to evaluate specific values of $h_{n}, h_{n}^{\prime}$, and $l_{n}^{\prime}$ for some positive real number $n$ by using the explicit relations established in Section 3 . To begin with, we show how to evaluate the values of $h_{2^{2 m}}$ and $h_{2^{2 m+1}}$ for every positive integer $m$. We only state the instances when $m=1,2,3$, and 4 .

Theorem 4.1. We have

(i) $h_{4}=1+\sqrt{2}-\sqrt{1+\sqrt{2}}$,

(ii) $h_{8}=\frac{\sqrt{2+\sqrt{2}}}{1+2^{1 / 4}}$,

(iii) $h_{16}=\frac{1+\sqrt{1+\sqrt{2}}}{\sqrt{1+\sqrt{2}}+(2(1+\sqrt{2}))^{1 / 4}}$,

(iv) $h_{32}=\frac{2^{1 / 4}+\sqrt{2}}{2^{5 / 8}+\sqrt{1+\sqrt{2}}}$,

(v) $h_{64}=\frac{2^{3 / 4}(1+\sqrt{2})^{1 / 4}+\sqrt{2(1+\sqrt{2})}}{1+2^{7 / 8}(1+\sqrt{2})^{3 / 8}+\sqrt{1+\sqrt{2}}}$,

(vi) $h_{128}=\frac{2^{7 / 8}+\sqrt{2+\sqrt{2}}}{1+2^{1 / 4}+2^{11 / 16}(2+\sqrt{2})^{1 / 4}}$,

(vii) $h_{256}=\frac{\sqrt{2}}{a+\sqrt{\sqrt{2}-a^{2}}}$,

(viii) $h_{512}=\frac{\sqrt{2}}{b+\sqrt{\sqrt{2}-b^{2}}}$,

where

$$
a=\frac{2^{3 / 4}(1+\sqrt{2})^{1 / 4}+\sqrt{2(1+\sqrt{2})}}{1+2^{7 / 8}(1+\sqrt{2})^{3 / 8}+\sqrt{1+\sqrt{2}}}
$$

and

$$
b=\frac{2^{7 / 8}+\sqrt{2+\sqrt{2}}}{1+2^{1 / 4}+2^{11 / 16}(2+\sqrt{2})^{1 / 4}} .
$$

Proof. For (i), letting $n=1$ in (3.2) and putting the value of $h_{1}=1$ from [7, Theorem 2.2], we find that

$$
\sqrt{2}\left(h_{4}+\frac{1}{h_{4}}\right)=h_{4}+2 .
$$

Solving the last equation for $h_{4}$ and using the fact that $h_{4}<1$, we complete the proof.

For (ii), letting $n=2$ in (3.2), putting the value of $h_{2}=\sqrt{2 \sqrt{2}-2}$ from [7, Theorem $4.7(\mathrm{i})$ ], we find that

$$
(-4+3 \sqrt{2}) h_{8}^{2}-4 \sqrt{-1+\sqrt{2}} h_{8}+2=0 .
$$

Solving (4.1) for $h_{8}$, and using the fact that $h_{8}<1$, we complete the proof. 
For (iii), letting $n=4$ in (3.2), putting the value of $h_{4}$ from the result of (i), solving for $h_{16}$, and using the fact that $h_{16}<1$, we complete the proof.

For (iv)-(viii), repeat the same argument as in the proof of (iii) to complete the proof.

See [7, Theorem 4.7] for different proofs of Theorem 4.1(i) and (ii). Hence $h_{2^{2 m}}$ and $h_{2^{2 m+1}}$ for $m=5,6,7, \ldots$ can be evaluated as in the proof of Theorem 4.1. We next evaluate the values of $h_{2^{2 m-2}}^{\prime}$ and $h_{2^{2 m-1}}^{\prime}$ for every positive integer $m$. We only state the instances when $m=1,2,3$, and 4 .

Theorem 4.2. Let $a$ and $b$ be as in Theorem 4.1. Then we have

(i) $h_{1}^{\prime}=\left(\frac{-1+\sqrt{2}}{2}\right)^{1 / 8}$,

(ii) $h_{2}^{\prime}=\frac{2^{1 / 16}}{(1+\sqrt{2})^{1 / 4}}$,

(iii) $h_{4}^{\prime}=\frac{(2(1+\sqrt{2}))^{1 / 16}}{(\sqrt{2}+\sqrt{1+\sqrt{2}})^{1 / 4}}$,

(iv) $h_{8}^{\prime}=\frac{2^{5 / 32}(1+\sqrt{2})^{1 / 8}}{\sqrt{1+2^{1 / 4}}}$

(v) $h_{16}^{\prime}=\left(\frac{1}{\sqrt{2} a^{2}}-\frac{1}{2}\right)^{1 / 4}$

(vi) $h_{32}^{\prime}=\left(\frac{1}{\sqrt{2} b^{2}}-\frac{1}{2}\right)^{1 / 4}$,

(vii) $h_{64}^{\prime}=\left(\frac{a^{2}\left(\sqrt{2}-a^{2}\right)}{2}\right)^{1 / 8}$,

(viii) $h_{128}^{\prime}=\left(\frac{b^{2}\left(\sqrt{2}-b^{2}\right)}{2}\right)^{1 / 8}$.

Proof. For (i), letting $n=4$ in (3.4) and putting the value of $h_{4}$ from Theorem 4.1(i), we find that

$$
(1+\sqrt{2}-\sqrt{1+\sqrt{2}})^{2}\left(2 h_{1}^{\prime 4}+1\right)=\sqrt{2} .
$$

Solving (4.2) for $h_{1}^{\prime}$ and using the fact that $h_{1}^{\prime}>0$, we complete the proof.

For (ii)-(viii), use (3.4) and Theorem 4.1(ii)-(viii), respectively, to repeat the same argument as in the proof of (i).

Next we show how to evaluate the values of $l_{2^{2 m}}^{\prime}$ and $l_{2^{2 m+1}}^{\prime}$ for every positive integer $m$. We only state the instances when $m=1,2,3$, and 4 .

Theorem 4.3. Let $a$ and $b$ be as in Theorem 4.1. Then we have

(i) $l_{4}^{\prime}=(2(1+\sqrt{2}))^{1 / 4}$,

(ii) $l_{8}^{\prime}=\sqrt{2+\sqrt{2}}$,

(iii) $l_{16}^{\prime}=1+\sqrt{1+\sqrt{2}}$

(iv) $l_{32}^{\prime}=\sqrt{4+3 \sqrt{2}+2 \sqrt{8+6 \sqrt{2}}}$,

(v) $l_{64}^{\prime}=\left(\frac{2 a^{4}}{\sqrt{2} a^{2}-1}\right)^{1 / 8}$, 
(vi) $l_{128}^{\prime}=\left(\frac{2 b^{4}}{\sqrt{2} b^{2}-1}\right)^{1 / 8}$,

(vii) $l_{256}^{\prime}=\left(\frac{2}{\sqrt{2} a^{2}-1}\right)^{1 / 4}$,

(viii) $l_{512}^{\prime}=\left(\frac{2}{\sqrt{2} b^{2}-1}\right)^{1 / 4}$.

Proof. For (i), letting $n=4$ in (3.6) and putting the value of $h_{4}$ from Theorem 4.1(i), we find that

$$
\left(\sqrt{2}(1+\sqrt{2}-\sqrt{1+\sqrt{2}})^{2}-1\right) l_{4}^{\prime 8}=2(1+\sqrt{2}-\sqrt{1+\sqrt{2}})^{4} .
$$

Solving (4.3) for $l_{4}^{\prime}$ and using the fact that $l_{4}^{\prime}>0$, we complete the proof.

For (ii)-(viii), use (3.6) and Theorem 4.1(ii)-(viii), respectively, to repeat the same argument as in the proof of (i).

See [8, Theorem 4.10] for different proofs of Theorem 4.3(i), (ii), and (iii).

\section{Evaluations of $J_{n}$}

We now turn to evaluations of the modular $j$-invariant in terms of $J_{n}$ in the case of when $n$ is of the form $n=2^{2 m-1}$ or $n=2^{2 m}$ for every positive integer $m$. We only show the instances when $m=1,2,3$, and 4 . Note that we have some new values of $J_{n}$ such as $J_{16}, J_{32}, J_{64}, J_{128}$, and $J_{256}$.

Theorem 5.1. Let $a$ and $b$ be as in Theorem 4.1. Then we have

(i) $J_{2}=\frac{5}{16}(-19+13 \sqrt{2})$,

(ii) $J_{4}=\frac{3}{32}(-724+513 \sqrt{2})$,

(iii) $J_{8}=\frac{5(83+58 \sqrt{2}+2 \sqrt{2(1423+1073 \sqrt{2})})}{16(8(5+4 \sqrt{2})+\sqrt{2(1817+1285 \sqrt{2})})}$,

(iv) $J_{16}=\frac{3(1+\sqrt{2})(731+540 \sqrt{2}-120 \sqrt{60+43 \sqrt{2}})}{2 \cdot 2^{3 / 4}\left(2+2^{3 / 4}\right)^{4}}$,

(v) $J_{32}=\frac{a^{8}-32\left(\sqrt{2}-a^{2}\right)^{2}\left(\sqrt{2} a^{2}-1\right)}{16 a^{16 / 3}\left(\sqrt{2}-a^{2}\right)^{2 / 3}\left(\sqrt{2} a^{2}-1\right)^{1 / 3}}$,

(vi) $J_{64}=\frac{b^{8}-32\left(\sqrt{2}-b^{2}\right)^{2}\left(\sqrt{2} b^{2}-1\right)}{16 b^{16 / 3}\left(\sqrt{2}-b^{2}\right)^{2 / 3}\left(\sqrt{2} b^{2}-1\right)^{1 / 3}}$,

(vii) $J_{128}=\frac{1-32 a^{2}\left(\sqrt{2}-a^{2}\right)\left(\sqrt{2} a^{2}-1\right)^{2}}{16 a^{2 / 3}\left(\sqrt{2}-a^{2}\right)^{1 / 3}\left(\sqrt{2} a^{2}-1\right)^{2 / 3}}$,

(viii) $J_{256}=\frac{1-32 b^{2}\left(\sqrt{2}-b^{2}\right)\left(\sqrt{2} b^{2}-1\right)^{2}}{16 b^{2 / 3}\left(\sqrt{2}-b^{2}\right)^{1 / 3}\left(\sqrt{2} b^{2}-1\right)^{2 / 3}}$.

Proof. For (i), letting $n=2$ in (1.13) and putting the values of $h_{1}^{\prime}$ from Theorem 4.2(i) and $l_{4}^{\prime}$ from Theorem 4.3(i), we complete the proof.

For (ii)-(viii), repeat the same argument as in the proof of (i) by using Theorems 4.2 and 4.3 .

See [6, Theorem 7.2.2] for alternative proofs of Theorem 5.1(i), (ii), and (iii). Observe that $J_{2^{2 m}}$ and $J_{2^{2 m+1}}$ for $m=5,6,7, \ldots$ can be evaluated by repeating the same argument as in the proof of Theorem 5.1. 
Corollary 5.2. The value of $j\left(\frac{3+\sqrt{-n}}{2}\right)$ can be evaluated in the case of when $n$ is of the form $n=2^{2 m-1}$ or $n=2^{2 m}$ for every positive integer $m$.

\section{References}

[1] B. C. Berndt, Ramanujan's Notebooks. Part V, Springer-Verlag, New York, 1998.

$[2]$ - Ramanujan's Notebooks. Part III, Springer-Verlag, New York, 1991.

[3] _ Number Theory in the Spirit of Ramanujan, American Mathematical Society, 2006.

[4] B. C. Berndt and H. H. Chan, Ramanujan and the modular j-invariant, Canad. Math. Bull. 42 (1999), no. 4, 427-440.

[5] D. A. Cox, Primes of the Form $x^{2}+n y^{2}$, Wiley, New York, 1989.

[6] J. Yi, The Construction and Applications of Modular Equations, Ph.D. Thesis, University of Illinois at Urbana-Champaign, 2001.

[7] — Theta-function identities and the explicit formulas for theta-function and their applications, J. Math. Anal. Appl. 292 (2004), no. 2, 381-400.

[8] J. Yi, Y. Lee, and D. H. Paek, The explicit formulas and evaluations of Ramanujan's theta-function $\psi$, J. Math. Anal. Appl. 321 (2006), no. 1, 157-181.

Dae Hyun Paek

Department of Mathematics Education

Busan National University of Education

BUSAN 611-736, KOREA

E-mail address: paek@bnue.ac.kr

JINHEE YI

Department of Mathematics and Computer Science

Korea Science Academy of KAIST

BUSAN 614-822, KOREA

E-mail address: jhyi100@kaist.ac.kr 